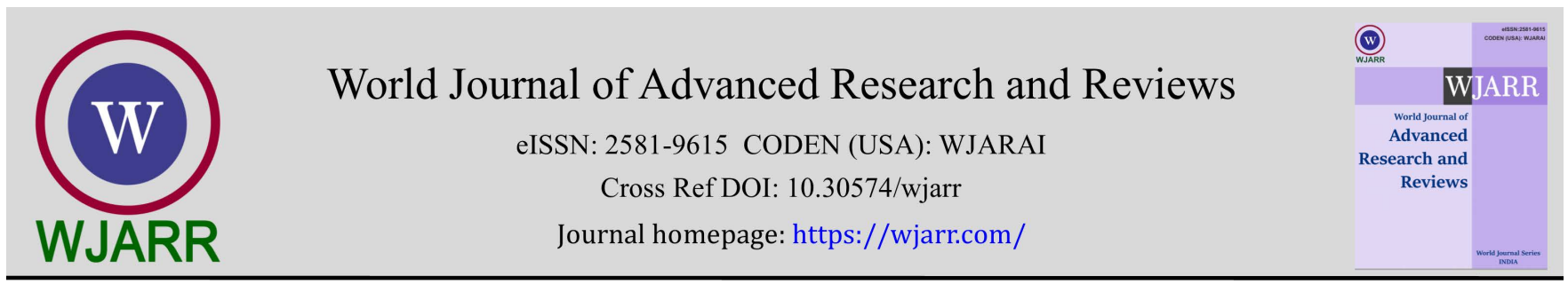

(REVIEW ARTICLE)

\title{
COVID-19 related challenges faced by Medical Laboratory Staff: A Review of Literature
}

\author{
Jignesh Sharma ${ }^{1}$ and Richard D. Nair ${ }^{2 *}$ \\ ${ }^{1}$ Medical Laboratory Scientist (WHO SSA), World Health Organization, Nadi, Fiji. \\ 2 School of Public Health and Primary Care, Fiji National University, Suva, Fiji.
}

World Journal of Advanced Research and Reviews, 2021, 12(02), 232-237

Publication history: Received on 06 October 2021; revised on 07 November 2021; accepted on 09 November 2021

Article DOI: https://doi.org/10.30574/wjarr.2021.12.2.0589

\begin{abstract}
Laboratory testing on the confirmation of COVID-19 results is an essential component and without the expertise of trained laboratory technicians this is not possible. The aim of this study was to review the impacts of COVID-19 on medical laboratory staff. The literature search was done using Medline, Embase, Scopus, and Proquest databases, and relevant keywords were applied to find studies which have been conducted in the field of Medical Laboratory Science specifically looking at the impacts on staff caused by the Covid-19 pandemic. All the studies pertaining to the topic published in 2020 and 2021 in English language were reviewed and the main themes were identified. The results showed that impacts of COVID-19 were felt by the staff, as they were pushed to their limits causing stress and burnout. Apart from this laboratory staff were faced with issues such as; shortage in terms of human resources, consumables, testing kits and reagents. This was an added factor to delays in testing and disruption to the testing Turnaround time (TATs) and also contributed to the stress and burnout of staff. Laboratory professionals and other health care staffs were pushed to the limits to ensure patient care was not affected and each patient was attended too without delay. Laboratory personnel's were pushed to their limits to ensure that test results were given on time.
\end{abstract}

Keywords: Impacts; COVID-19; Medical laboratory staff; Literature review

\section{Introduction}

The coronavirus disease 19 (COVID-19) is a highly transmittable and pathogenic viral infection caused by severe acute respiratory syndrome coronavirus 2 (SARS-CoV-2), which emerged in Wuhan, China and spread around the world. The intermediate source of origin and transfer to humans is not known, however, the rapid human to human transfer has been confirmed widely. There is no clinically approved antiviral drug or vaccine available to be used against COVID-19. However, few broad-spectrum antiviral drugs have been evaluated against COVID-19 in clinical trials, resulted in clinical recovery [1].

The health system of many countries has been overwhelmed by the pandemic, with many losing a significant number of their health professionals in the fight against the virus. While doctors and nurses are so visible at the front lines and are being applauded for the gallant role, they are playing in the recovery of hundreds of thousands of COVID-19 patients, there is little about those behind their successes, the Medical laboratory scientists (MLS). Medical laboratory science is the bedrock of diagnostic medicine and the role of the MLS in containing any pandemic cannot be overemphasized [2].

An effective and timely diagnostics approach is fundamental and successful containment of any outbreak is ineffective without MLS at the fore-front of this. They are the ones testing clinical specimens from infected and clinically recovered patients. As disease detectives, their role in the fight against the COVID-19 pandemic; include is not limited to: diagnosis,

\footnotetext{
${ }^{*}$ Corresponding author: Richard D Nair

School of Public Health and Primary Care, Fiji National University, Suva, Fiji.
}

Copyright (C) 2021 Author(s) retain the copyright of this article. This article is published under the terms of the Creative Commons Attribution Liscense 4.0. 
monitoring, confirmation of recovery, safety and efficacy testing of broad-spectrum antiviral agents, discovery and development of vaccines, validation of testing protocols and testing kits, offering of advisories to guide government policy on containment at all levels amongst others [2].

Care of Mental health in frontline medical workers around the world is urgently needed. The frontline workers undergo great psychological stress and are faced with many challenges and losses. The transmission of COVID-19 from human to human and the increasing number of deaths could elicit their unease and fear about getting infected. The bad outcomes of some critical patients, the suffering of patients and their relatives could lead to worsening anxiety and mental distress. The lack of medical supplies, the uncertain information from various resources, the loneliness and worries for their loved ones may increase the risk of existing mental pressure. These factors will increase medical staffs' anxiety and cause reluctance to work [3].

Furthermore, laboratory testing not only plays an essential role in confirming the diagnosis of COVID-19 and detecting infected individuals but also has encountered enormous challenges in combating this rapid-spreading and lifethreatening disease, Apart, from this they assist in containment strategies and also aid in implementation of new policies and guidelines [4]. The aim of this review is to gather information on the impact of COVID-19 on medical laboratory staff and the importance of laboratory staff during this pandemic. Apart from this, the study will also see how the pandemic has affected the laboratory staff both physiologically and psychologically something that has also affected the dental professionals who are actively engaged in the COVID-19 response [5].

\section{Methodology}

This literature review focused on several aspects related to the impact of COVID-19 on medical laboratory staff. Four databases were used to search for publications on relevant studies: Medline, Embase, Scopus, and ProQuest. The keywords used included: (Impact OR influence) AND (COVID-19 OR coronavirus) AND ("Clinical Laboratory Staff" OR "Medical laboratory scientists" AND ("Laboratory services" OR Lab). The focus of the search were studies published between 2020 and 2021 in English language. Duplicated studies from different databases were excluded. The titles of all the studies were scanned by two independent researchers and those not relevant were excluded. The abstracts of the remaining studies were reviewed and the full text of the 20 articles that met the study inclusion criteria were printed for future review and to formulate the themes that are discussed below.

\subsection{Findings}

This review found three themes that determine the impact of COVID - 19 on medical laboratory staffs that are: Mental health and its consequence, Turnaround Time (TAT) of other tests, Lack of resources, technic and protocol of COVID test

These themes are presented and discussed below:

\subsubsection{Theme 1: Mental health and its consequence}

The COVID pandemic is associated with both psychological and physiological effects on the health of medical laboratory staff. Due to the increasing demands in testing numbers and less human resources, staff are left with no choice but to put themselves at risk [6]. Starting its spread in 2019, COVID-19 affected China and spread all over the world in a short period. Facing this dramatic emergency, many countries implemented extraordinary measures to limit viral transmission. In this critical situation everyone's life changed due to restrictions of movement and social contacts, in particular, Health Care Workers (HCWs) uninterruptedly continued to work in such critical situations, with the risk of being affected by COVID-19; therefore, they might be considered as one of the most vulnerable categories of health professionals to develop psychological stress and other mental health symptoms [6]. Stress classically refers to "the bodily processes that result from circumstances that place physical or psychological demands on an individual's"; although a certain degree of stress can facilitate task performance, it becomes problematic when the demands outweigh the perceived resources to cope with [6].

The adverse impact of the COVID-19 pandemic has a direct effect on the sleep quality and anxiety levels among laboratory staff, this reinforces the need for urgent attention to sleep disorders and mental health globally. Laboratory staff are particularly exposed to higher levels of stress and work demand. Additionally, HCWs, specifically laboratory staff have a higher risk of contamination when compared to the other health cadres. A study by Drager at el [7] showed that 1,817 out of $4,384(41.4 \%)$ laboratory staff presented new-onset of insomnia during the pandemic or worsening of preexisting insomnia. This sample size allowed $95 \%$ significance level with a margin of error of just $1.46 \%$. The majority of participants (61.4\%) described that their sleep quality worsened during the pandemic. Anxiety is a well-known risk 
factor for insomnia and has been identified in up to $44.7 \%$ of HCWs during the COVID-19 pandemic. While new-onset of burnout was also an independent predictor of worsening preexisting insomnia, burnout was reported by $20.1 \%$ of the participants and by $30.7 \%$ of those complaining of insomnia [7].

Medical personnel, including laboratory staff have been found to be more psychologically traumatized and experience higher levels of stress, depression, and anxiety. This can be explained by the expected anxiety and fear of getting infected due to their risk of exposure and worry of infection transmission to their families, friends, or colleagues. This puts them at a great challenge of balancing between professional duty, humanity, and personal fear for oneself and others, a situation that can often cause conflict and dissonance in many laboratory personnel. Apart from exposure, health workers suffer from panic and worry of getting sick or dying, feeling of helplessness, or face blame from others who are ill, all precipitating a state of mental collapse. Different presentations of psychiatric illnesses have been found, ranging from depression, anxiety, panic attacks, somatic symptoms, and post-traumatic stress disorder symptoms to delirium, psychosis, and even suicide. These symptoms have been associated with a younger age and increased feelings of selfguilt; stigmatization, and social avoidance [8].

A high prevalence of symptoms of post-traumatic stress, anxiety and depression were identified in health professionals including hyper arousal, anger, loss of motivation at work, difficulty concentrating and trouble falling asleep. Individuals with high levels of resilience have less irritability, less concern for environmental stimuli, better interpersonal relationships, fewer headaches, musculoskeletal pains and lower levels of depression. If these symptoms persist over time, the feeling of a lack of control and uncertainty at work increases, leading to burnout among the laboratory personnel's during COVID -19 [9]. These syndromes are work related and due to the COVID-19 pandemic it has become more evident among laboratory personnel's. It is characterized by high emotional exhaustion, high levels of depersonalization and low personal accomplishment. Lab personnel may experience dysphoric symptoms, such as tiredness or emotional exhaustion. The symptoms appear in relation to work situations in individuals who previously did not show psychosocial alterations. Additionally, burnout is associated with a decrease in work performance due to negative behaviors towards work. In particular, in health workers exposed to traumatic situations during this pandemic, the presence of burnout has been detected, as well as a reduction in the ability to apply coping strategies or negative attitudes towards work. In addition to the symptoms of exhaustion, related to anxiety, depression or other symptoms related to physical pathologies (e.g., cardiovascular problems), burnout can lead to intention to leave the post, which would incur high costs [9].

Moreover, a recent report by health authorities of Iran mentioned that around 10,000 HCWs have contracted COVID-19 and at least 43 are known to have died of this disease [10]. This high rate of infection and mortality has a tremendous impact on the healthcare system. Therefore, this pandemic is expected to have substantial psychological impact on HCWs. Burnout can have serious consequences for both patients and the HCWs. It not only results in poor physical and mental health outcomes, lack of motivation, absenteeism, and low morale in the staff, but also leads to deterioration of the quality of care provided by the affected staff with resulting poor outcomes for patients [11].

The fact that laboratory staff are at the most risk of work-related exposure to COVID-19, work-related exposure can occur anytime in the workplace, during work-related travel to an area with local community transmission, as well as on the way to and from the workplace. In the same work setting, there may be jobs with different levels of risk, and different jobs or work tasks may have similar levels of exposure. Some workers may be at higher risk of developing severe COVID19 illness because of age or pre-existing medical conditions [12]. This increased risk to exposure and the staff burnout due to the increased work load there has been tremendous staff turnovers; which may be due to the fear among laboratory staff of being infected because of their pre-existing conditions or because due to the lack of adequate personnel protective equipment (PPE) and also due to the fact that during the pandemic staff members were either deployed to other centers to help cope with the increasing work load or the staff members were in quarantine [3].

\subsubsection{Theme 2: Turn Around Time (TAT) of other tests affected due to COVID-19}

The importance of the laboratory diagnosis of the infection caused by severe acute respiratory syndrome coronavirus 2 (SARS-CoV-2), has seen the Turnaround Times (TAT) of other laboratory test to have been affected and there are delays caused in giving out results. The preferred testing method is the real-time Reverse Transcription-PCR (RT-PCR) test similar to that developed for the diagnosis of SARS-CoV-2, which roughly takes around 4 to 8 hours of processing time [13]. Apart from real-time RT-PCR test, Cartridge-based diagnostic often allows the (i) diagnosis of critically ill cases in a short time period, (ii) assessment of suspected patients very rapidly, allowing for a specific epidemiological management, and (iii) transfer diagnostics to point-of-care scenarios including smaller laboratories. Rapid testing has been shown to provide important information immediately, improving patient management, and the test performance of the new Xpert Xpress SARS-CoV-2 cartridge assay which roughly takes 30 to 45 minutes of processing time [14]. 
COVID-19 is difficult to diagnose, and a delay between the onset of symptoms and accurate diagnosis is frequently observed. It is worth noting that, except reported confirmed cases of pneumonia, there are still many undiagnosed and delayed-diagnosis infections due to lack of diagnostic reagents for the virus and the long waiting time for diagnosis. In reality, only a proportion of symptomatic patients can be diagnosed in time, while the rest may be delayed due to limited hospital or diagnostic resources. The intermittent increase of medical resources makes the actual diagnosis efficiency different. In resource-poor setting such as developing nations and smaller pacific islands, the infectious individuals have to wait for longer time for diagnosis due to limited amount of diagnostic equipment's and low diagnostic efficiency of the test kits etc. The waiting time for delayed diagnosis is critical for disease transmission and control [15].

Due to the rapid transmission of SARS-CoV-2, the role of diagnostic testing is dependent on the types of test available, the resources required for testing, and the time to obtain results. In other words, the rapid identification of suspected cases remains a high priority regardless of the circumstances of low allocated resources and less human resources. Furthermore, reverse Transcriptase-Polymerase Chain Reaction (rRT-PCR) requires professionally trained staff to operate sophisticated laboratory facilities, which are usually located at a central laboratory (biosafety level 2 or above), and is often time-consuming, requiring from few hours up to 2 or 3 days to obtain laboratory results. This often leaves a rapidly rising number of potential cases untested and thus opening a gaping hole in SARS-CoV-2 prevention efforts. Furthermore, this time-consuming process of sample testing is not only extremely disadvantageous but also unsafe since the virus needs to be contained [16].

A study done by Lapić at el., [17] in Croatia, showed that the urgent and somehow haphazard reorganization of the Emergency Department (ED) during the COVID- 19 pandemic requiring a temporary shutdown of the (ED)-caused laboratories to significantly prolonged overall TAT. The first underlying reason is undoubtedly the need for longer specimen transport to the relatively distant central laboratory, done exclusively on foot, extending the specimen transportation time from a previous 10 minutes up to a median of 52 minutes. Simultaneously, as the extraordinary pandemic situation required staff redistribution to newly established duties such as patient triage and monitoring at several hospital entrances, the ED experienced acute staff shortages. Additional quick and efficient workers urgently needed for laboratory specimen transportation tasks were simply not available because of staff redistribution and inadequate human resource. In addition, the specimens from the ED were processed within the central laboratory together with the usual emergency inpatient specimens, effectively doubling its workload and thus surpassing the current capacity of centrifuges and analyzers. This in turn caused a less prominent but still detectable prolongation of TAT for ED specimens [17].

\subsubsection{Theme 3: Lack of resources, technic and protocol of COVID test}

The sudden widespread of the COVID-19 pandemic has placed medical triage decision-making in the spotlight. Lifesaving ventilators have become scarce, clinicians are being forced to conserve and use resources wisely in even the wealthiest countries. The pervasiveness of air travel and high rate of transmission has caused this pandemic to spread swiftly throughout the world. Ethical triage decisions are commonly based on the utilitarian approach of maximizing total benefits and life expectancy. HCWs are faced with an epic proportion of medical and ethical decisions regarding allocation of these precious resources [18].

Emerging viral pandemics can place extraordinary and sustained demands on public health and health systems and on providers of essential community services. Such demands will create the need to ration medical equipment and come up with interventions. Rationing has already started in the United States (US), perhaps the earliest example was the near-immediate recognition that there were not enough high filtration N-95 masks for health care workers, prompting contingency guidance on how to reuse masks designed for single use [23]. Physicians in Italy have proposed directing crucial resources such as intensive care beds and ventilators to patients who can benefit most from the treatment. The rapidly growing imbalance between supply and demand for medical resources in many countries presents an inherently normative question: How can medical resources be allocated fairly during a Covid-19 pandemic? This issue is not just limited to the developing nation where health care is not at its best state but also seen in developed countries where best health care services are provided. From the lack of testing kits and reagents to less human resource has really put pressure on laboratory professionals and HCWs to rethink their resource sourcing options. Resources are prioritized for COVID patients and COVID based test and to those staff who are in close contact with COVID patients [19].

It has become imperative for developing nation's laboratories to ensure that their testing is not compromised and that quality results are given after a particular test is performed. Real-time rRT-PCR based assays performed on respiratory specimens remain the gold standard for COVID-19 diagnostics. However, point-of-care technologies and serologic immunoassays are rapidly emerging with high sensitivity and specificity as well. Even though excellent techniques are available for the diagnosis of symptomatic patients with COVID-19 in well-equipped laboratories as critical gaps still 
remain in screening asymptomatic people who are in the incubation phase of the virus, as well as in the accurate determination of live viral shedding during convalescence to inform decisions for ending isolation [16]. Infectious diseases, such as the most recent case of coronavirus disease 2019, have brought the prospect of Point-Of-Care (P0C) diagnostic tests into the spotlight. A rapid, accurate, low-cost, and easy-to-use test in the field could stop epidemics before they develop into full-blown pandemics. Unfortunately, despite all the advances, it still does not exist [20].

Concerns about ongoing spread of disease from individuals who are infected without symptoms have been raised. Efforts to develop and implement testing protocols are underway, and expanded testing for COVID-19 is a necessary immediate step towards understanding and resolving this crisis. As tests become more available, observing principles of evidence-based clinical reasoning concerning the meaning of diagnostic test results is essential. For negative test results in particular, failure to do so has direct implications for the safety of the public and health care workers and for the success of efforts to curb the pandemic. Specifically, anticipation of a less-visible second wave of infection from individuals with false-negative test results is needed. The diagnostic sensitivity of RT-PCR testing for other viruses is highly variable, but early data from China suggested relatively poor sensitivity of initial RT-PCR tests. Even with sensitivity values as high as $90 \%$, the magnitude of risk from false negative test results will be substantial as testing becomes more widespread as the prevalence of COVID-19 infection rises. False-negative results are consequential. Individuals with these results may relax physical distancing and other personal measures designed to reduce the transmission of the virus to others [21].

The COVID-19 pandemic poses a major global challenge, with a massive yet possibly underestimated burden and several unknowns with a subtle clinical presentation and asymptomatic carriage, and in the absence of specific treatment and vaccines, it is clear that an early and accurate diagnosis is crucial for the control of the disease. Although rt-PCR represents a cornerstone for SARS-CoV-2 laboratory diagnosis, several limitations have been observed. Therefore, a combined approach harmonizing laboratory methods (ie, rt- PCR and serology) with imaging features and clinical findings is essential to guide patient management and infection control [22]. The ongoing, unprecedented outbreak of COVID-19 globally has emphasized the importance of the laboratory diagnosis of human coronavirus infections in order to limit the spread as well as to appropriately treat those patients who have a serious infection. These ShortTurnaround-Time (STAT) tests will be very important for real-time patient management and infection control decisions, especially when other less infectious forms of pneumonia are present and respiratory isolate resources are scarce. These assays are safe, simple, and fast and can be used in local clinics and hospitals that already have the needed instruments and that are responsible for identifying and treating such patients [13].

\section{Conclusion}

It can be said that due to COVID-19 pandemic HCWs as a whole were globally affected. A major global challenge was brought forward to relook at how medical procedures and test were carried out. A shift in the paradigm was seen especially in the medical laboratory field, as test numbers increased and workload accelerated which led to shortage of medical equipment's, human resource, testing reagents and kits to widespread death of medical staff and the general population. Laboratory professionals and other health care staffs were pushed to the limits to ensure patient care was not affected and each patient was attended too without delay. Laboratory personnel's were pushed to their limits to ensure that test results were given on time. Due to the increased testing numbers and staff burnout, there were staff burnout which greatly affected the TATs of other test, however prioritization was given to COVID-19 testing and other test were also carried out to ensure no patient was left behind.

\section{Compliance with ethical standards}

\section{Disclosure of conflict of interest}

The authors declare no conflict of interest.

\section{References}

[1] Shereen M, Khan S, Kazmi A, Bashir N, Siddique R. COVID-19 infection: Emergence, transmission, and characteristics of human coronaviruses. Journal of Advanced Research. 2020; 24: 91-98.

[2] Ibeh, Isaiah, Enitan, Samson, Richard, Akele, Isitua, Chinwe. A Review of the COVID-19 Pandemic and the Role of Medical Laboratory Scientists in containment. 2020; 30: 68-89. 
[3] Huang J, Liu F, Teng Z, Chen J, Zhao J, Wang X et al. Care for the Psychological Status of Frontline Medical Staff Fighting Against Coronavirus Disease 2019 (COVID-19). Clinical Infectious Diseases. 2020; 71(12): 3268-3269.

[4] Fang B, Meng Q. The laboratory's role in combating COVID-19. Critical Reviews in Clinical Laboratory Sciences. 2020; 57(6): 400-414.

[5] Gohil D, Nair R, Mohammadnezhad M, Raman Reddy K. Impact of COVID- 19 on the Psychological Health of Dental Professionals: A Systematic Review. Global Journal of Health Science. 2021; 13(7): 1.

[6] Babore A, Lombardi L, Viceconti M, Pignataro S, Marino V, Crudele M et al. Psychological effects of the COVID2019 pandemic: Perceived stress and coping strategies among healthcare professionals. Psychiatry Research. 2020; 293: 113-366.

[7] Drager L, Pachito D, Moreno C, Tavares A, Conway S, Assis M, et al. Sleep Disturbances, Anxiety, and Burnout during the COVID-19 Pandemic: a nationwide cross-sectional study in Brazilian Healthcare Professionals. 2020.

[8] Elamin M, Hamza S, Abdalla Y, Mohammed Mustafa A, Altayeb M, Mohammed M, et al. The Psychological Impact of the COVID-19 Pandemic on health professionals in Sudan 2020. Sudan Journal of Medical Sciences. 2020; 5470

[9] Luceño-Moreno L, Talavera-Velasco B, García-Albuerne Y, Martín-García J. Symptoms of Posttraumatic Stress, Anxiety, Depression, Levels of Resilience and Burnout in Spanish Health Personnel during the COVID-19 Pandemic. International Journal of Environmental Research and Public Health. 2020; 17(15): 5514.

[10] Ing E, Xu A, Salimi A, Torun N. Physician Deaths from Corona Virus Disease (COVID-19). 2020.

[11] Jalili M, Niroomand M, Hadavand F, Zeinali K, Fotouhi A. Burnout among healthcare professionals during COVID19 pandemic: a cross-sectional study. 2020.

[12] Adb.org. 2021 [Internet] [cited 10 November 2021]. Available from: https://www.adb.org/sites/default/files/publication/614811/safety-well-being-workers-communities-covid19.pdf

[13] Tang Y, Schmitz J, Persing D, Stratton C. Laboratory Diagnosis of COVID-19: Current Issues and Challenges. Journal of Clinical Microbiology. 2020; 58(6).

[14] Goldenberger D, Leuzinger K, Sogaard K, Gosert R, Roloff T, Naegele K et al. Brief validation of the novel GeneXpert Xpress SARS-CoV-2 PCR assay. Journal of Virological Methods. 2020; 284: 113925.

[15] Rong X, Yang L, Chu H, Fan M. Effect of delay in diagnosis on transmission of COVID-19. Mathematical Biosciences and Engineering. 2020; 17(3): 2725-2740.

[16] Younes N, Al-Sadeq D, AL-Jighefee H, Younes S, Al-Jamal O, Daas H, et al. Challenges in Laboratory Diagnosis of the Novel Coronavirus SARS-CoV-2. Viruses. 2020; 12(6): 582.

[17] Lapić I, Komljenović S, Knežević J, Rogić D. COVID-19 Pandemic Once Again Exposes the Weakest Link in Laboratory Services: Specimen Delivery. Laboratory Medicine. 2020; 51(6): e83-e86.

[18] Solnica A, Barski L, Jotkowitz A. Allocation of scarce resources during the COVID-19 pandemic: a Jewish ethical perspective. Journal of Medical Ethics. 2020; 46(7): 444-446.

[19] Emanuel E, Persad G, Upshur R, Thome B, Parker M, Glickman A, et al. Fair Allocation of Scarce Medical Resources in the Time of Covid-19. New England Journal of Medicine. 2020; 382(21): 2049-2055.

[20] Zhu H, Zhang H, Ni S, Korabečná M, Yobas L, Neuzil P. The vision of point-of-care PCR tests for the COVID-19 pandemic and beyond. TrAC Trends in Analytical Chemistry. 2020; 130: 115984.

[21] West C, Montori V, Sampathkumar P. COVID-19 Testing. Mayo Clinic Proceedings. 2020; 95(6): 1127-1129.

[22] Russo A, Minichini C, Starace M, Astorri R, Calò F, Coppola N. Current Status of Laboratory Diagnosis for COVID19: A Narrative Review. Infection and Drug Resistance. 2020; 13: 2657-2665.

[23] Centers for Disease Control and Prevention (CDC). 2021 [Internet] [cited 4 November 2021]. Available from: https://www.cdc.gov/coronavirus/2019-ncov/hcp/respirators-strategy/index.html. 